\title{
Fluorescent Specimen Preparation Techniques for Confocal Microscopy
}

\author{
Judith A. Drazba
}

Imaging Facility, The Cleveland Clinic Foundation, 9500 Euclid Avenue, Cleveland, OH 44195

Every good microscopist knows that specimen preparation is critical for obtaining good images. Unfortunately some people think that a confocal microscope can make poorly stained specimens look good. A confocal makes images sharper by rejecting out-of-focus light but it cannot discriminate between specific and non-specific staining or between a specific probe and an autofluorescent background. It takes a picture of all the light coming from a selected focal plane. If anything, a confocal will render artifacts sharper and more beautiful. Therefore, good staining protocols and adequate controls are more important than ever.

Confocal microscopes require specimens to be either reflective or fluorescent. Since biological cells and tissues are not very reflective we use fluorescent molecules that can either be tagged onto biological macromolecules to show their specific cellular location (i.e. antibodies) or bind to specific cellular components (i.e. dyes). Until recently the most frequently used fluorophores have been FITC, which appears green when excited, and TRITC, which appears red. Despite their utility, these molecules come with problems such as only getting to see them for seconds to minutes before they are irretrievably bleached. Today's fluorescent molecules come in "designer" colors that can be better separated based on their emission wavelengths for multiple labeling and are much brighter and more stable than their older cousins. The advent of improved antiphotobleach mounting media now allows us to view specimens for hours and keep them viable in storage for years.

Dye selection The first decision in developing a staining protocol is what colors to use. If your specimen has considerable autofluorescence you'll need to determine what wavelength range is the most offensive and then use a fluorophore as far away from that range as possible. If you're labeling multiple molecules in the same sample you'll want to choose fluorophores whose emission profiles are sufficiently non-overlapping that you can separate them well in the microscope, but also have the best excitation profile for the available confocal light sources, usually lasers. For multiple labeling you also need to minimize cross-reactivity by carefully choosing the animal sources of the antibodies to which the probes are conjugated. Organelle specific dyes in combination with an antibody can tell you if your molecule of interest is associated with a particular cellular component.

Fixation and Permeabilization Appropriate fixation is critical to good staining. Avoid glutaraldehyde if possible. Even small quantities produce intense autofluorescence that will interfere with visualizing your probe. Quenching protocols can help, but it's best avoided. Paraformaldehyde is the better alternative. If you're using fluorescent dyes be sure to buy those that are "fixable" because, unlike antibodies, some can only be used on live cells and tissues. Most antibody staining is done on fixed tissue, which often requires permeabilization. A frequently asked question in confocal microscopy is: "How thick can my sample be?" The appropriate question in response is: "How far can you get your antibody to penetrate into the tissue?" In most instances that distance is less than the working distance of most objective lenses. The porosity of the tissue, the length of staining and the concentration of detergent will all play a role. Collection of confocal optical sections through the specimen will quickly reveal the success or failure of the staining (Fig 1). Analysis of an "XZ" section, available on some microscopes, is an even better indicator of stain penetration (Fig 2). 
Quantity vs. Quality Titering of antibody or dye concentration is very important. In the case of antibodies, too much is as bad as too little. Over saturation can actually decrease signal due to steric hindrance and will substantially increase non-specific binding even if there is adequate blocking. Cellular components can appear very convincingly stained this way. To have any chance of interpreting the results it is very important to have controls that have not seen any antibody as well as controls that have only been exposed to secondary antibodies. Signal strength will also be determined by the method of antibody application and should be matched to the prevalence of the molecule being probed. Antibodies that are directly conjugated to a fluorochrome may produce less background, but since there's little amplification you may not be able to see molecules that exist in low quantity. Sandwich techniques work better in these cases either with fluorescently labeled secondary antibodies or with biotinylated secondaries and fluorescently labeled avidin.

Future Trends The advent of fluorescent proteins is a recent solution to the antibody penetration problem. Through the magic of molecular biology specific molecules can be tagged and then endogenously expressed by cells so that the specimen comes virtually "pre-labeled." Other techniques such as fluorescent in-situ hybridization are providing valuable information, but also require careful preparation for reliable results.

\section{References:}

1. R. Bacallao et al.,Handbook of biological Confocal Microscopy, Plenum, New York, 1995, J.B. Pawley (ed.)

2. R. Bacallao, EH Stelzer, Preservation of biological specimens for observation in a confocal fluorescence microscope. Methods Cell Biol 1989; 31:437-52
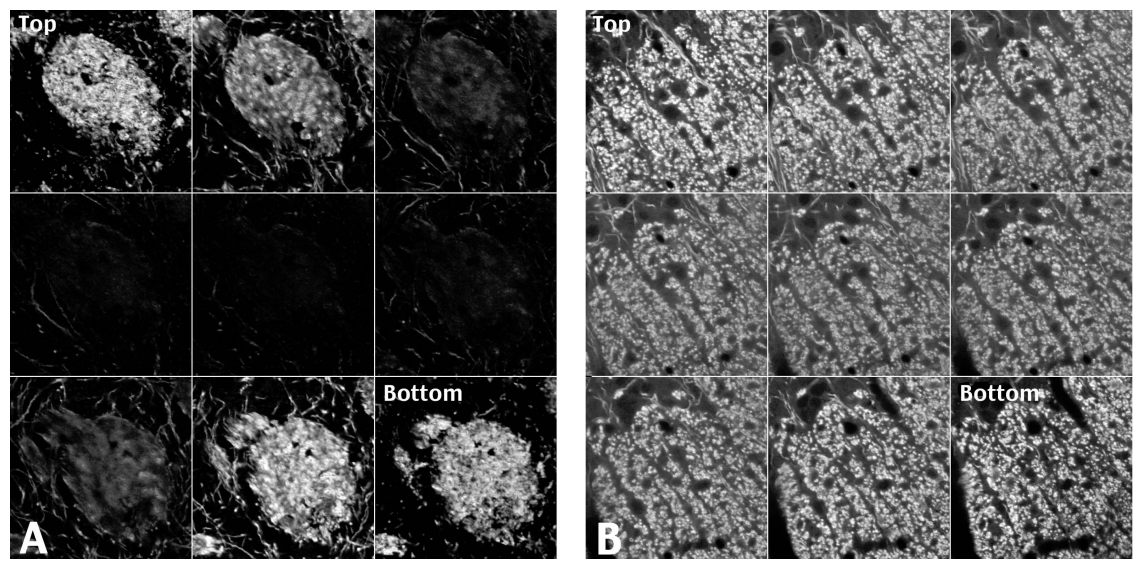

Figure 1. Series of optical sections collected with a Leica-TCS-NT confocal laser scanning microscope comparing antibody penetration into $30 \mu \mathrm{m}$ thick free-floating sections of rat brain permeabilized with either (A) $0.5 \%$ or (B) $10 \%$ Triton-X 100 detergent.
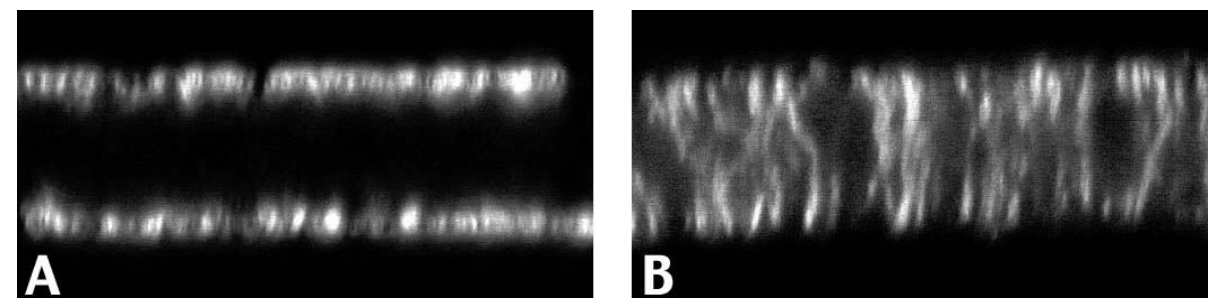

Figure 2. Same specimen shown in Figure 1 imaged in cross-section (XZ slice). 